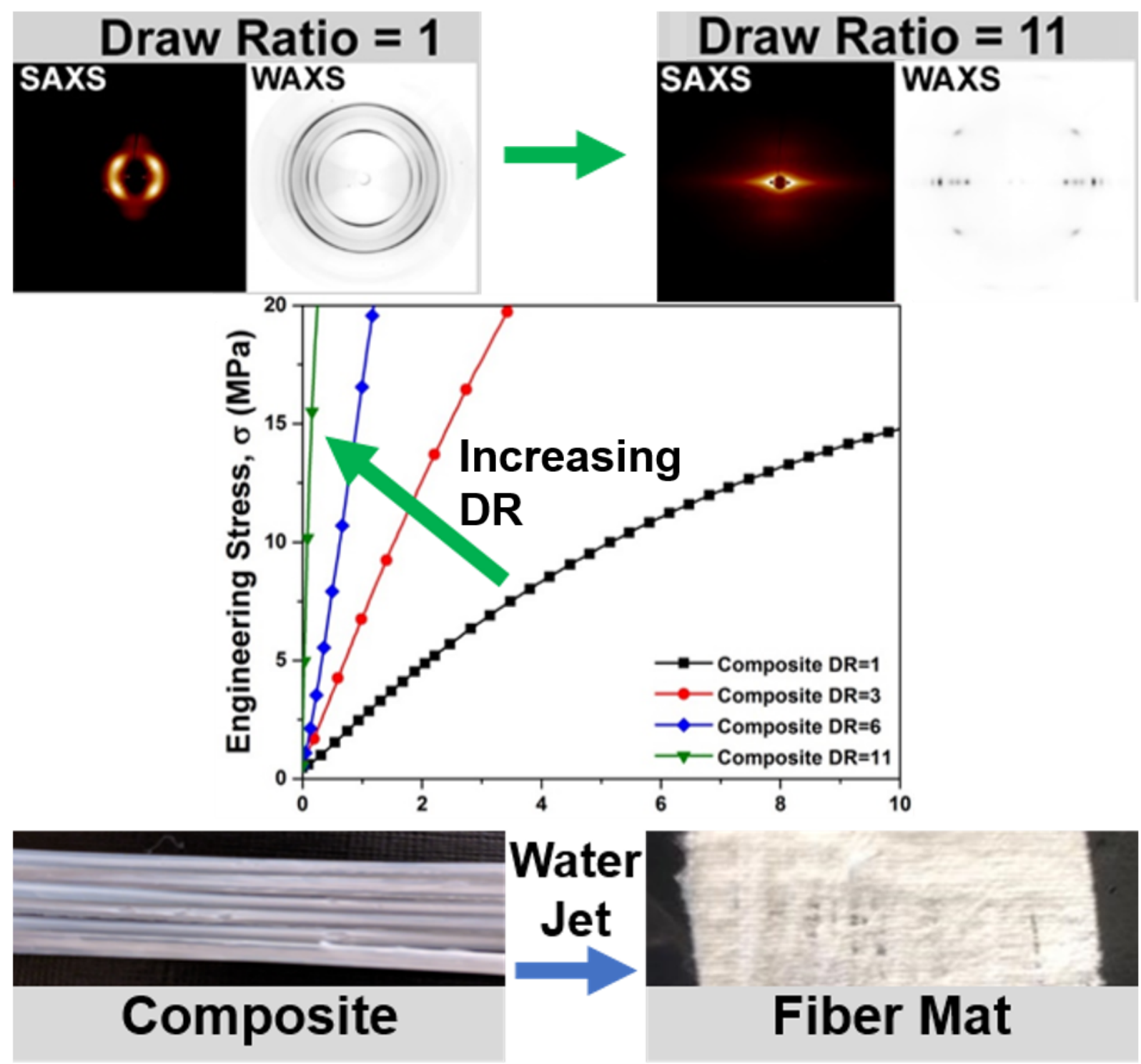




\title{
Mechanically Tunable Dual-Component Polyolefin Fiber Mats via Two-Dimensional Multilayer Coextrusion
}

\author{
William R. Lenart ${ }^{\mathrm{a} \dagger}$, Keon-Soo Jang ${ }^{\mathrm{a} \dagger}$, Alex M. Jordan ${ }^{\mathrm{a}}$, Eric Baer ${ }^{\mathrm{a}}$, LaShanda T.J. Korley ${ }^{\mathrm{a},{ }^{*}}$ \\ ${ }^{\mathrm{a} C e n t e r}$ for Layered Polymeric Systems, and Department of Macromolecular Science and \\ Engineering, Case Western Reserve University, 2100 Adelbert Rd, Cleveland OH, 44106 \\ *Author to whom all correspondence should be addressed, lashanda.korley@case.edu. \\ † Co-first authors
}

\begin{abstract}
Recently developed two-dimensional multilayer coextrusion and post-process drawing were combined to fabricate polyolefin composites with independently tunable size (9 width x 3 thickness $\mu \mathrm{m}$ to 5 width $\times 0.9$ thickness $\mu \mathrm{m}$ ) and mechanics (e.g. modulus from 1340 to 2010 $\mathrm{MPa}$ ). Post-process drawing of the composite, studied via in situ synchrotron WAXS and SAXS, imparted higher crystallinity and more uniform and aligned crystallites $\left(f_{H, P P}=0.93\right.$ and $f_{H, H D P E}=$ 0.90) resulting in an improved modulus, while also increasing and narrowing the melting temperature. After drawing, the composites were simultaneously delaminated and consolidated using a high pressure water jet to produce dual-component fiber mats with high specific surface area, which was related to the fiber size and rectangular cross-section unique to this process. The tunability of the HDPE and PP fibers produced via this process hold unique advantages over solvent-based techniques, such as electrospinning, for many high performance applications.
\end{abstract}

\section{INTRODUCTION}

Commodity polyolefins, such as polyethylene and polypropylene, occupy a dominate position in today's plastics market with polyethylene alone capturing a market share of $34.8 \%$ in the US during 2014. [1] These polymers enjoy their market position because of their remarkable diversity of applications and properties derived from the methods used to synthesize and process the materials. [2] Applications for polyolefins range from food packaging to complex biomedical devices. Additionally, these polymer feedstocks are extremely cheap, making them ideal for a variety of disposable applications and rapid market penetration. Polyethylene is one of the most versatile polymers on the market, which, depending on the chain structure and molecular weight, can be used to make products such as grocery bags, filters, milk jugs, and orthopedic implants. Polypropylene, being more rigid and thermally resistant, fills many of the needs polyethylene products alone cannot, such as carpeting or battery separators.

Until recently, it has been extraordinarily challenging to process these materials into ultrafine fibers, a size scale necessary for a variety of high performance applications, most prominently ultrafiltration. These applications require non-wovens with extremely small fiber sizes to reduce pore size, and, in the case of air filtration, the higher surface area drastically improves particle loading. [3-5] Current melt-based polyolefin fiber processing techniques, such as melt blowing, typically have a minimum fiber size of one to two microns. [6-9] If the polymer is too viscous, the melt strength limits the fiber size and material selection. 
Solution electrospinning, wherein a polymer solution is ejected through a charged needle onto a substrate, is another technique for the production of nanoscale fibers. Fiber size and quality in electrospinning is tuned via the interplay of solvent volatility, solution polarity, solution viscosity, potential, and collection method. [10-20] There has been considerable activity in this field with significant success for a wide range of polymers, excluding polyolefins. Although it is possible to fabricate electrospun polyolefin fibers, the spinning conditions are extremely harsh, requiring high temperatures and solvents like xylene; in addition, only extremely low throughputs are achieved for these polyolefin systems, limiting industrial relevance. [21-23] The emerging technique of melt electrospinning has overcome some of the current obstacles of traditional melt processing and solution electrospinning by using high electric potentials over short distances to produce fibers on the order of $300 \mathrm{~nm}$, but is still challenged by limited throughput. [24]

Recently, the melt coextrusion process has combined concepts of horizontal and vertical layer multiplication to produce an extruded polymer-polymer composite with tunable domain sizes, which are controlled by the degree of process multiplication, exit die configuration, take-off method, and post-process drawing. After a suitable separation process, such a water jetting or solvation, fibers with submicron dimensions approaching the nanoscale are obtained from these extruded composites. Early efforts in this area have demonstrated its material flexibility, focusing on the manufacture of fibrous systems derived from polymers, such as poly( $\varepsilon$-caprolactone) (PCL), poly(amide-6) (PA6), and poly(ethylene terephthalate (PET). [25] When combined with postprocess orientation, this scalable and modular process overcomes many limitations of both conventional melt fiber processing techniques and electrospinning. In general, extruded submicron fibers may be obtained from two immiscible polymers with a similar viscosity at a temperature in the range of $180-300{ }^{\circ} \mathrm{C}$.

Melt-based techniques in general eliminate the use of volatile solvents, while the coextruded fiber approach allows more consistent control of fiber sizes compared to melt blowing, melt spinning, or rotary jet spinning. [6, 24, 26, 27] The continuous nature of the fiber forming process allows for convenient post-process fiber drawing and orientation, which reduces fiber size, while simultaneously improving mechanical strength and crystallinity. Increased crystallinity enhances the overall robustness of the fibrous mat not only by improving the mechanical properties but also by increasing the chemical resistance and narrowing the melting temperature. $[25,28]$ Recently, we optimized the removal of the poly(ethylene oxide) (PEO) matrix from PEO/PCL single fiber component composites and the subsequent uniaxial drawing of the PCL fibers. We fabricated PCL fibers with similar size and thermo-mechanical properties as electrospun PCL fibers, but the extruded fibers exhibited a substantial 6-fold increase in specific surface area and the ability to tune both fiber size and mechanical properties. [28] Other research efforts have focused on the surface functionalization of these PCL extruded fibers with biologically relevant moieties, such as RGD and IKVAV peptides, with an eye towards biomedical applications. [2932]

In this work, we fabricated dual component HDPE/PP fiber mats with tunable fiber size, specific surface area, and mechanical properties. We explored the structural development within these polyolefin composites to probe the interplay of crystallinity, fiber size, and mechanical properties. This dual component system, from a scalable manufacturing process, demonstrates the design of ultra-fine polyolefin fibers within areas such as ultrafiltration and battery separation. 


\section{EXPERIMENTAL}

Materials

High density polyethylene (HDPE, Dow Chemical Elite G5960), polypropylene (PP, Dow Chemical D207.1), and polystyrene (PS, American Styrenics 685D) were used to produce the polymer-polymer composites in this study. PS was used as the sacrificial separating layer because of its brittleness, low cost, and expected poor adhesion to both HDPE and PP. The most suitable extrusion conditions were obtained by determining the temperature at which HDPE, PP, and PS exhibit a similar viscosity match by measuring the melt flow index (MFI, Galaxy I Model D7054 Kayeness Inc.). A viscosity match was found at $240{ }^{\circ} \mathrm{C}$ (Figure S1).

\section{Fiber Composite Processing}

The coextrusion process has been described in great detail elsewhere for horizontal multilayering, vertical multi-layering, as well as the combination of the two to fabricate extruded fiber composites. [33-38] A brief summary is provided for this specific system. Three extruders were employed to produce the coextruded HDPE/PP/PS composites (Scheme 1). The first two extruders combined the flow of HDPE/PP, constituting the fiber-forming components, at $240{ }^{\circ} \mathrm{C}$. The size ratio of the two fiber forming domains were controlled with the competing pump rates from the first two extruders, set at a 1:1 ratio each using 15 rotations per minute (RPM). Rather than exit the feedblock horizontally, as in typical multi-layering, the flow was rotated. A series of multiplying dies then split and stacked the flow of HDPE/PP into a number of layers $2^{n}$, where $n$ is the number of multipliers. After the vertical multiplication, the flow traveled through the feedblock of the third extruder, operating at 3 RPM, where a sacrificial PS "skin" layer enveloped the vertical layers perpendicularly. The flow was further multiplied as previously reported, but now the 'layers' are horizontal. The overall number of individual components in the final composite followed as $2^{n}$ with a total $2^{m}$ horizontal layers and each fiber forming layer containing $2^{n-m}$ layers, where $n=15$ and $m=6$ are the number of multiplying dies before and after the second feedblock, respectively. Following horizontal layer multiplication, the flow was forced through a tape die (12.5 mm width by $0.5 \mathrm{~mm}$ height orifice) onto a variable speed chill roll at $90{ }^{\circ} \mathrm{C}$ as the take-off method. The final extruded composite displayed a cross-sectional structure comprised of a two-dimensional array of alternating PS separating layers as the matrix and HDPE/PP vertical layers as the fiber-forming domains. HDPE and PP comprised $91 \mathrm{v} / \mathrm{v} \%$ of the overall composite with PS as the remaining $9 \mathrm{v} / \mathrm{v} \%$. A single HDPE fiber composite was also manufactured under the same conditions using PS as both the sacrificial matrix and the second fiber forming domain. Fabrication of HDPE single-component fiber mats was challenged by incomplete PS removal and premature fiber fracture. 


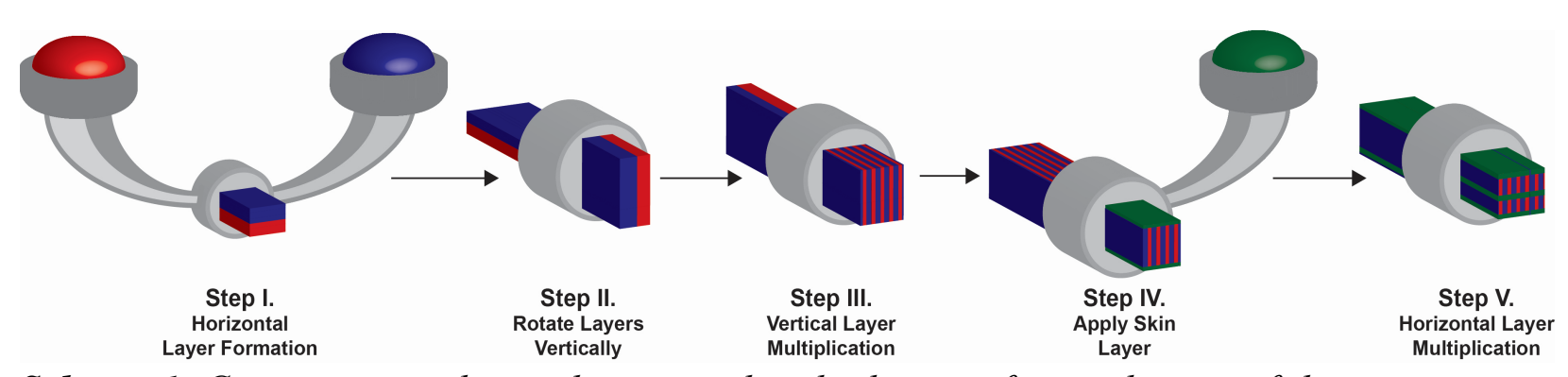

Scheme 1. Coextrusion with two-dimensional multiplication for production of three component coextruded composites. HDPE (red), PP (blue), and PS (green). Adapted with permission from ref. [50].

\section{Uniaxial Drawing (Orientation)}

Composites were cut to a length of $30 \mathrm{~mm}$ and placed between polyurethane grips on a Zwick/Roell mechanical tester with a gauge length of $10 \mathrm{~mm}$. The furnace temperature was set to $105^{\circ} \mathrm{C}$, and the composite was allowed to equilibrate for one hour. Using a temperature above the glass transition temperature $\left(\mathrm{T}_{\mathrm{g}}\right)$ of PS $\left(102{ }^{\circ} \mathrm{C}\right.$, Figure S2), PS phases of the composite were easily drawn, while maintaining overall composite integrity. Composites were drawn at a constant velocity of $50 \mathrm{~mm} / \mathrm{min}$ to the desired draw ratio (DR) up to 11 and held under tension with ambient cooling to $25^{\circ} \mathrm{C}$.

\section{In Situ SAXS/WAXS}

X-ray studies were conducted at Brookhaven National Laboratory (BNL) National Synchrotron Light Source - I (NSLS-I) at the Advanced Polymers Beamline, X27C, using a radiation wavelength $\lambda=0.1371 \mathrm{~nm}$. The drawn composites were oriented with the fiber-like HDPE and PP domains perpendicular to the X-ray beam, such that the fiber-axis and stretching direction coincided. Two-dimensional X-ray patterns were recorded on a Brüker Smart $1500 \mathrm{X}$ ray $\mathrm{CCD}$ detector $(1024 \times 1024$ pixels $)$ at each integer draw ratio up to $\mathrm{DR}=11$, which corresponded to an engineering strain of $1000 \%$. The wide-angle X-ray scattering (WAXS) sample-to-collector distance of $146 \mathrm{~mm}$ was calibrated using an $\mathrm{Al}_{2} \mathrm{O}_{3}$ standard with a 104 reflection at $2 \theta=21.14^{\circ}$. The small-angle X-ray scattering (SAXS) sample-to-collector distance of $1783 \mathrm{~mm}$ was calibrated using a silver behenate (AgBe) standard with a 001 reflection at a scattering vector $(q)$ of $1.076 \mathrm{~nm}^{-1}$. X-ray patterns were collected with an exposure time of two minutes to ensure adequate exposure, while simultaneously minimizing stress relaxation effects within the composite samples. Both SAXS and WAXS patterns were corrected for the background incoherent air scattering and detector dark field by collecting background and dark field patterns for two minutes to match the sample exposure time. The background and dark field images were subtracted from each SAXS and WAXS image and corrected for the transmission factor of the HDPE/PP/PS composite.

SAXS profiles were integrated radially along $q$ and azimuthally averaged over a range $30^{\circ}<\varphi<30^{\circ}$ to yield a meridional one-dimensional (1D) profile of intensity as a function of $q$. To obtain an equatorial SAXS profile of intensity as a function of $q$, radial integrations along $q$ were azimuthally averaged over a range $60^{\circ}<\varphi<120^{\circ}$. WAXS profiles were first integrated radially along $2 \theta$ to obtain $1 \mathrm{D}$ profiles of intensity as a function of Bragg angle.

It is possible to use previously defined relationships between the c-axis of a crystallite and two reflections along the $h k 0$ layer line to quantify the degree of crystalline orientation for PP and HDPE using Hermans orientation function, Equation 1. 


$$
\begin{gathered}
f_{H}=\frac{3\left\langle\cos ^{2} \varphi\right\rangle_{c}-1}{2} \\
\left\langle\cos ^{2} \varphi\right\rangle_{c, P P}=1-0.901\left\langle\cos ^{2} \varphi\right\rangle_{040, P P}-1.099\left\langle\cos ^{2} \varphi\right\rangle_{110, P P} \\
\left\langle\cos ^{2} \varphi\right\rangle_{c, H D P E}=1-0.555\left\langle\cos ^{2} \varphi\right\rangle_{200, H D P E}-1.445\left\langle\cos ^{2} \varphi\right\rangle_{110, H D P E}
\end{gathered}
$$

WAXS profiles were azimuthally integrated over a range $0^{\circ}<\varphi<90^{\circ}$. Since the $h k 1 \mathrm{PP}$ reflections coincide with the $110 \mathrm{HDPE}$ reflection, it is not possible to use the $110 \mathrm{HDPE}$ reflection in this calculation. By assuming that the azimuthal intensity distribution is similar for the 200 and 110 crystallographic planes, Equation 2b simplifies to Equation $\mathbf{2 c}$ where the average cosine angle term is given by Equation 3.

$$
\begin{gathered}
\left\langle\cos ^{2} \varphi\right\rangle_{c, H D P E}=1-2\left\langle\cos ^{2} \varphi\right\rangle_{200, H D P E} \\
\left\langle\cos ^{2} \varphi\right\rangle=\frac{\int_{0}^{\frac{\pi}{2}} I(\varphi) \sin \varphi \cos ^{2} \varphi d \varphi}{\int_{0}^{\frac{\pi}{2}} I(\varphi) \sin \varphi d \varphi}
\end{gathered}
$$

\section{Differential Scanning Calorimetry}

Differential scanning calorimetry (DSC) measurements were recorded on a Q100 (TA Instruments). The samples were ramped from $0{ }^{\circ} \mathrm{C}$ to $200{ }^{\circ} \mathrm{C}$ at a heating rate of $3{ }^{\circ} \mathrm{C} / \mathrm{min}$ using 3-5 $\mathrm{mg}$ of material sealed in hermetic aluminum pans. Only the first heating was used to observe the effect of DR on the crystalline structure in the HDPE and PP domains of the composite. After correcting the heating scan for instrument baseline fluctuations, integrations were taken of the melting endotherm of HDPE and PP to determine the melting enthalpy $\left(\Delta H_{f}\right)$ of each domain. The melting temperature $\left(\mathrm{T}_{\mathrm{m}}\right)$ was determined from the respective endotherm maximum. The nominal flow rates of the extrusion system (45.5:45.5:9 by volume HDPE:PP:PS) were used to convert the $\Delta H_{f}$ from composite weight to individual component weight.

\section{Composite Tape Tensile Testing}

Composite tapes were cut to $40 \mathrm{~mm}$ with $10 \mathrm{~mm}$ of the composite placed in both the top and bottom grip to yield a $20 \mathrm{~mm}$ gauge length. The tensile samples were elongated at a constant rate of $100 \% \mathrm{~min}^{-1}$, and the stress response recorded using a Zwick/Roell (100 N load cell) mechanical tester. The Young's modulus (Ет) of each composite sample was determined using the linear stress vs. strain region at low strain $(0.05-0.25 \%$ strain) via a linear regression analysis. A minimum of five samples per DR were tested to ensure an appropriate consistency between samples. To determine the effective modulus $\left(\mathrm{EF}_{\mathrm{F}}\right)$ of the fiber-like HDPE and PP domains within the composite, a volume additive model was used (Equation 4). (39)

$$
\begin{gathered}
E_{T}=E_{F} V_{F}+E_{P S} V_{P S} \\
E_{F}=\frac{E_{T}+E_{P S} V_{P S}}{1-V_{P S}}
\end{gathered}
$$

The Young's modulus of the PS (Eps) phase can be measured at various DRs, and the effective volume fraction of PS (VPS) was 0.09 as obtained from the nominal extrusion flow rates. 


\section{$\underline{\text { Delamination }}$}

Due to the brittle nature of PS and poor adhesion of all three polymers, it was possible to separate the coextruded composite into its components using a continuously moving, high pressure water jet (Atomizing Systems Inc.). A pressure of $11.0 \mathrm{MPa}$ with a nozzle diameter of $0.25 \mathrm{~mm}$ $(470 \pm 35 \mathrm{~mL} / \mathrm{min})$ was used for a single coextruded composite tape to fracture and remove the PS while simultaneously separating the HDPE and PP fiber-forming components (Scheme 2). After delamination, the resultant material is a mixture of HDPE and PP fibers with rectangular crosssections.

To overcome the difficulties of testing the mechanical properties of a single fiber, the composites were simultaneously delaminated and consolidated into a hydroentagled mat. To minimize fiber damage, mats were fabricated using a wire mesh screen. Two types of square wire mesh were utilized: (1) 16 mesh - wire diameter of $0.4572 \mathrm{~mm}$, an opening width of $1.1176 \mathrm{~mm}$, and an open area of $50.7 \%$, and (2) 30 mesh -wire diameter of $0.3048 \mathrm{~mm}$, an opening width of $0.5613 \mathrm{~mm}$, and an open area of $40.8 \%$. Twenty drawn fiber composites were assembled onto a steel plate and secured with tape for each fiber mat produced. The 16 mesh wire was placed onto the fiber composites bundle, and $5 \mathrm{~min} / \mathrm{cm}^{2}$ of water jetting was applied to each side. The 16 mesh was then replaced with the 30 mesh for another $5 \mathrm{~min} / \mathrm{cm}^{2}$ of water jetting for each side.

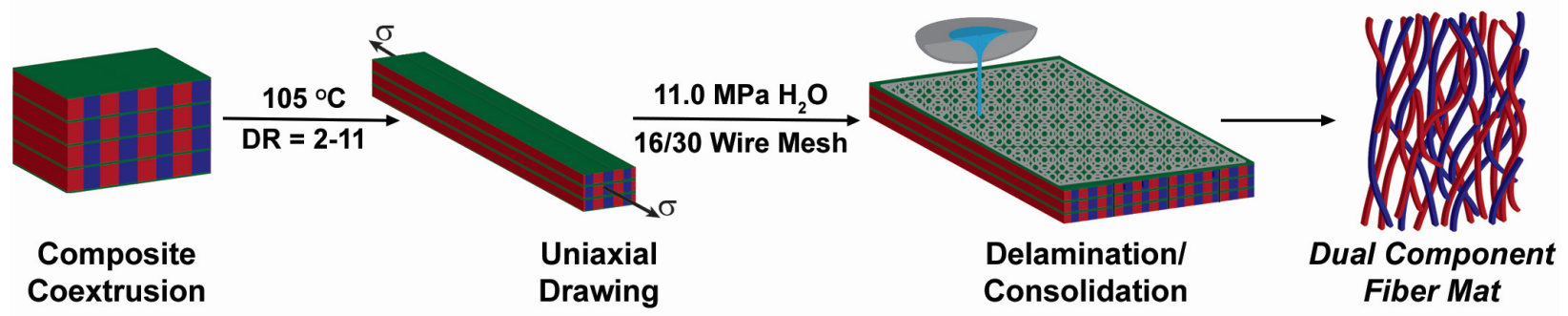

Scheme 2. Uniaxial drawing of and delamination/consolidation the HDPE/PP/PS extruded composite. HDPE (red), PP (blue), and PS (green). Adapted with permission from ref. [50].

\section{Residual PS Characterization}

Fourier transform infrared (FT-IR) spectroscopy was utilized to qualitatively determine the residual PS content in the HDPE/PP fibers. A dilute solution (in chloroform; Sigma-Aldrich, 99\%) of each sample was cast onto a $\mathrm{NaCl}$ pellet and allowed to completely dry for ten minutes. Thirtytwo individual scans were recorded under a nitrogen atmosphere using a Bio-rad Excalibur Series spectrometer equipped with a mercury-cadmium-telluride detector.

${ }^{1} \mathrm{H}$ NMR spectra were acquired (in $\mathrm{CDCl}_{3}$; Cambridge Isotope Laboratories, Inc., 99.8\%) using a Varian 600-MHz spectrometer to quantitatively determine the residual PS content. The normalized PS content was determined based on integration of the aromatic proton shift (6.30-7.20 $\mathrm{ppm})$ and the chloroform reference peak $(7.26 \mathrm{ppm})$. The integrated area of the coextruded composite before delamination was normalized to the nominal PS composition of $9 \mathrm{v} / \mathrm{v} \%$. This value was then used to compare the removal of PS during delamination at various water jetting times.

\section{Surface Area Analysis}

The surface area of the delaminated fibers was measured with a Micromeritics Tristar II surface area analyzer using multi-point Brunauer-Emmett-Teller (BET) theory to calculate the surface area from Krypton adsorption isotherms after degassing for $12 \mathrm{hrs}$ under nitrogen at $70^{\circ} \mathrm{C}$ 
to minimize moisture and air. [40] The weight of each sample for measurement after degassing was ca. $100 \mathrm{mg}$.

\section{Surface Morphology Analysis}

After delamination, scanning electron microscopy (SEM, JEOL JSM-6510LV) was used to determine the average fiber size (50 width and 50 thickness measurements). The fibers were sputter-coated with $15 \mathrm{~nm}$ of gold to minimize fiber movement and surface charging during imaging. The magnification used was in the range of x100-2000 with an electron beam voltage of $15 \mathrm{kV}$.

\section{Fiber Mat Tensile Testing}

Uniaxial tensile testing was conducted with the same Zwick/Roell (100 N load cell) mechanical tester utilized for composite analysis. After consolidation, the modulus of each mat was determined by uniaxially drawing at $100 \% \mathrm{~min}^{-1}$ under ambient conditions with a $10 \mathrm{~mm}$ gauge length and an approximate cross-section of $5 \mathrm{~mm} \times 0.5 \mathrm{~mm}$. The modulus was determined from the linear elastic region between $0.05-0.25 \%$ strain. For each fiber mat, the length, width, and thickness, and mass was measured. A calculated apparent density was then compared to the theoretical density of a solid blend of 50/50 (v/v) HDPE/PP film to obtain the void fraction. The void fraction is used to calculate the cross sectional area of the fibers under load to determine stress.

\section{$\underline{\text { Results and Discussion }}$}

\section{Fiber Composite Characterization}

Dual-component polyolefin fibers were fabricated using a recently developed process of multilayer coextrusion and subsequent delamination. We explored the ability to draw and orient the coextruded composites prior to delamination to facilitate uniform deformation of the fibrous domains. The connection between crystal structure evolution and ultimate fiber size/mechanics is critical to the rapid development of these polyolefin fibers for high performance applications.

Following coextrusion, the 3-component HDPE/PP/PS composites were drawn uniaxially to reduce the HDPE and PP domain sizes as well as to impart chain orientation within the domains. The evolution of the chain orientation during uniaxial drawing was probed using synchrotron radiation. WAXS experiments provided information regarding short length scale periodicity and crystallite orientation, while SAXS studies examined long-range order of the extruded $\mathrm{HDPE} / \mathrm{PP} / \mathrm{PS}$ composites. 


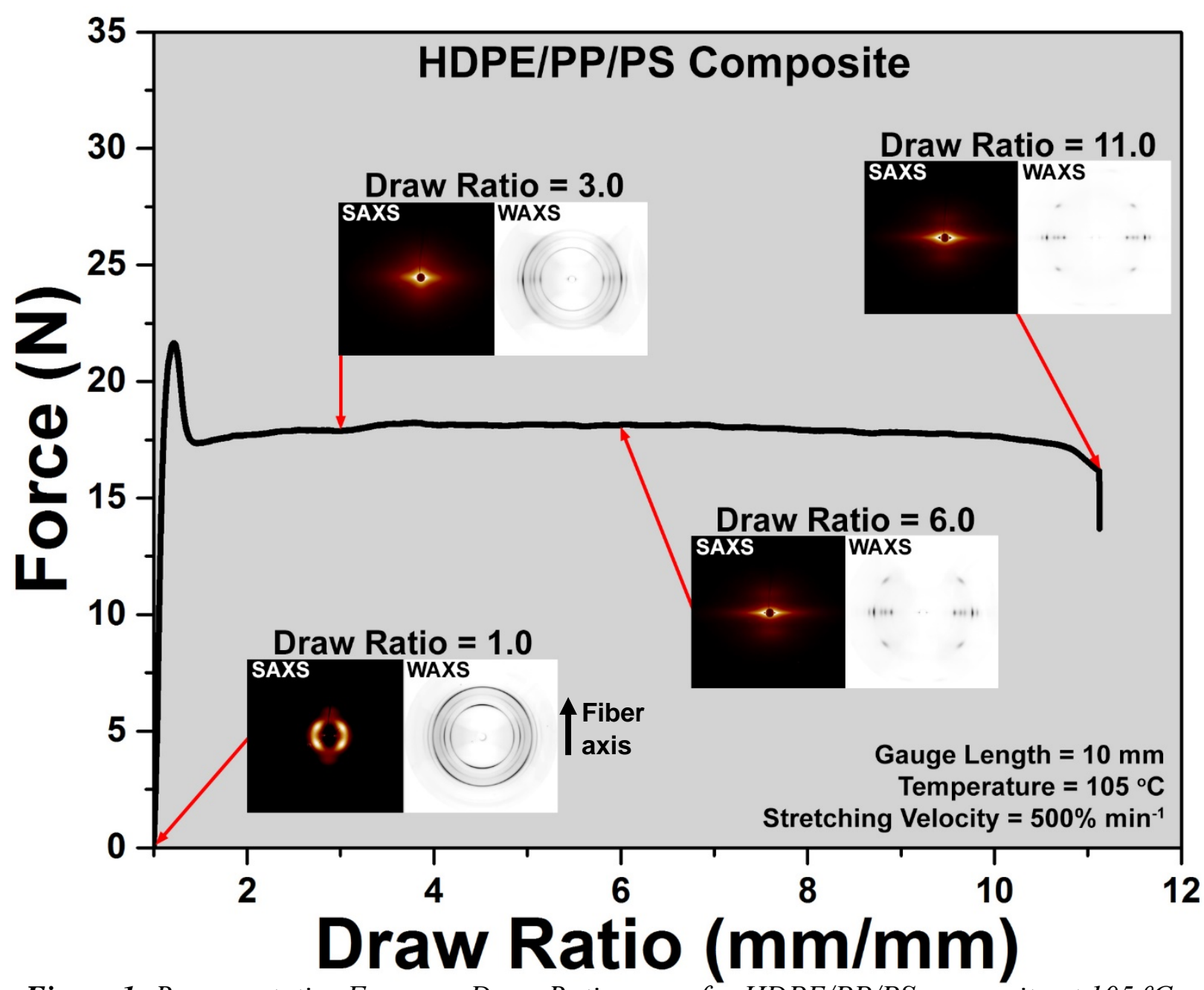

Figure 1: Representative Force vs. Draw Ratio curve for $H D P E / P P / P S$ composite at $105^{\circ} \mathrm{C}$, $500 \% \mathrm{~min}^{-1}$ strain rate along with selected $2 D$ SAXS (black background) and WAXS (white background) profiles.

During uniaxial drawing, the crystal structure of the HDPE and PP domains shifted from a spherulitic crystal structure to a stacked lamellae crystal structure (Figure 1). The 4-point pattern of the SAXS profile at DR $=1$ indicated that either the HDPE or PP domains contain spherulitic crystallites. Once the sample reached $\mathrm{DR}=3$, the 4-point pattern transitioned to a meridional bar above and below the equator, indicating that the spherulites transitioned to stacked folded-chain lamellae. The strong equatorial reflection observed at $\mathrm{DR}=3$ may be attributed to two phenomena: stress-induced microvoids, indicated by a maximum at $0.26 \mathrm{~nm}^{-1}$, or stress-induced crystalline fibers. [41] However, the lack of a defined maxima suggests the formation of stress-induced crystalline fibers, but the opaque nature of the composite inhibited examination via polarized optical microscopy.

In addition to restructuring of the crystallites in the HDPE and PP domains, the WAXS patterns revealed that alignment also occurred. The isotropic ring-like reflections $(\mathrm{DR}=1)$ narrowed to point-like reflections up to $\mathrm{DR}=6$. Six reflections were observed for the monoclinic structure of PP: three crystallographic planes along the hk0 layer line corresponding to the 110 , 040, and 130 planes, one crystallographic plane along the hk2 layer line corresponding to the 022 plane, and two coincidental reflections, 041 and 111. The orthorhombic structure of HDPE 
exhibited only two crystallographic reflections on the $h k 0$ layer line, corresponding to the 110 and 200 planes. The average relative alignment between the c-axis of the crystallites and the fiber direction provides a measure of the degree of orientation, which is quantified by the Hermans orientation function $\left(f_{H}\right)$. Values for $f_{H}$ range from -0.5 (c-axis perpendicular to the fiber axis) to 1 (crystallites completely parallel to the fiber), where 0 indicates random orientation. [42, 43]

The 110 and 040 reflections of PP were used to calculate the Hermans orientation function as there was no interference with any other crystallographic planes; only the 200 reflection for HDPE was utilized (Figure 2a). The as-extruded composite at room temperature had a $f_{H, P P}=$ 0.04 and $f_{H, H D P E}=0.10$, confirming random orientation of crystallites as expected from a spherulitic crystal structure. Upon heating, the crystallite orientation remained unchanged. However, uniaxial drawing up to $\mathrm{DR}=6$ induced an increase to $f_{H, P P}=0.93$ and $f_{H, H D P E}=0.90$, which changed only slightly up to DR $=11$ (Figure $2 \mathbf{b}$ ).
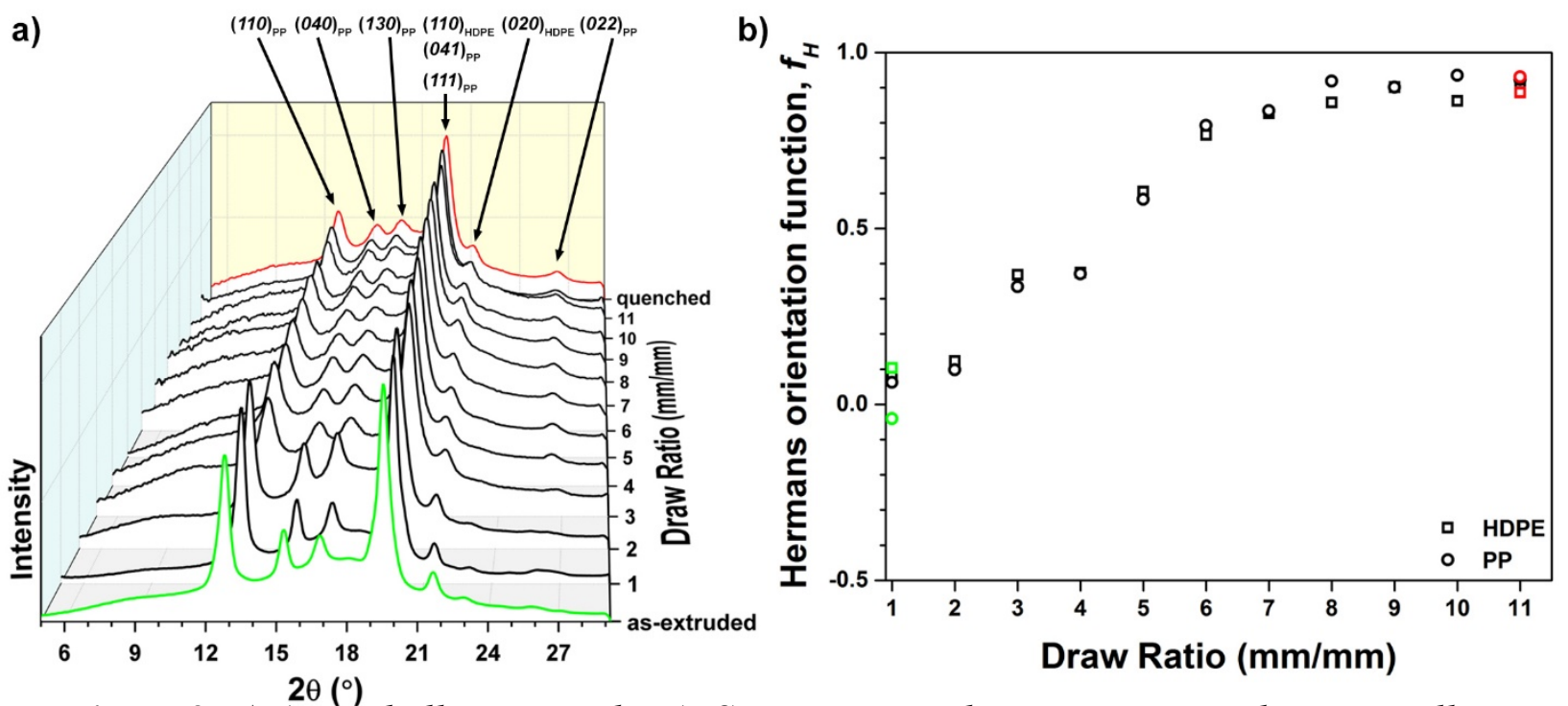

Figure 2. a) Azimuthally averaged WAXS integrations showing HDPE and PP crystalline reflections; b) $f_{H} v$ s. Draw Ratio for HDPE and PP crystallites.

As the crystallites restructured and oriented, the crystallinity increased along with the melting temperature of HDPE (Table 1). The increasing melting enthalpy of both HDPE and PP confirms the evidence of strain-induced recrystallization, which was observed as an equatorial streak in the SAXS pattern. Using $293.6 \mathrm{~J} / \mathrm{g}$ as the $\Delta H_{f}$ for $100 \%$ crystalline HDPE, the crystallinity of HDPE increased slightly from $28.1 \%$ to $33.0 \%$ at $\mathrm{DR}=6$. [44] Since the breadth of the melting endotherm decreased while the melting temperature increased (Figure 3), the change in HDPE crystallinity was attributed to the crystallites increasing in thickness and uniformity. [45] The crystallinity increased even more significantly for PP, rising from $9.7 \%$ to $23.9 \%$ when using 207 $\mathrm{J} / \mathrm{g}$ as the $\Delta H_{f}$ for $100 \%$ crystalline PP. [46] However, the breadth of the melting endotherm decreased without a corresponding increase in melting temperature, indicating more PP crystallites and greater uniformity. [47] 


\begin{tabular}{|c|c|c|c|c|}
\hline $\begin{array}{c}\text { DR } \\
(\mathrm{m} / \mathrm{m})\end{array}$ & $\begin{array}{c}\text { HDPE } \\
\Delta \mathbf{H}_{\mathrm{f}} \\
(\mathrm{J} / \mathrm{g})\end{array}$ & $\begin{array}{c}\text { HDPE } \\
\mathbf{T}_{\mathbf{m}} \\
\left({ }^{\circ} \mathbf{C}\right)\end{array}$ & $\begin{array}{c}\mathbf{P P} \\
\Delta \mathbf{H}_{\mathbf{f}} \\
(\mathbf{J} / \mathbf{g})\end{array}$ & $\begin{array}{c}\mathbf{P P} \\
\mathbf{T}_{\mathrm{m}} \\
\left({ }^{\circ} \mathbf{C}\right)\end{array}$ \\
\hline 1 & 82.5 & 130 & 20.1 & 167 \\
\hline 2 & 84.6 & 132 & 25.8 & 168 \\
\hline 3 & 87.8 & 136 & 32.5 & 168 \\
\hline 4 & 89.4 & 136 & 38.6 & 168 \\
\hline 5 & 89.1 & 137 & 34.2 & 168 \\
\hline 6 & 96.8 & 136 & 49.4 & 167 \\
\hline 7 & 97.6 & 137 & 45.6 & 168 \\
\hline 8 & 95.8 & 137 & 45.2 & 168 \\
\hline 9 & 96.0 & 137 & 43.6 & 167 \\
\hline 10 & 95.1 & 136 & 46.2 & 168 \\
\hline 11 & 93.6 & 137 & 49.2 & 168 \\
\hline
\end{tabular}

We investigated the relationship between the observed changes in crystallinity and crystal structure of the HDPE/PP/PS composites and the mechanical response of the composite. Four draw ratios $(1,3,6$, and 11) were chosen to represent this structural evolution. (Figure 4a). As expected, the spherulitic crystal structure of the composite at $\mathrm{DR}=1$ exhibited the lowest modulus due to the isotropic distribution of the component lamellae within the spherulitic crystals, but displayed the highest extensibility. The higher extensibility may be attributed to the break-up of the spherulites into its component lamellae and the isotropic nature of the chains within the amorphous regions. Upon orientation and subsequent chain alignment, an increase in modulus and a decrease in strain-at-break were noted for $\mathrm{DR}=3$ and 6 (Figure 4a). Interestingly, the extensibility continued to decrease upon further drawing $(\mathrm{DR}=11)$, while the modulus remained relatively constant (Figure 4c). The increasing modulus up to a plateau corresponded very well with the stable crystallinity values of both phases, while the decreasing extensibility was due to the amorphous chain alignment in the direction of uniaxial drawing. 


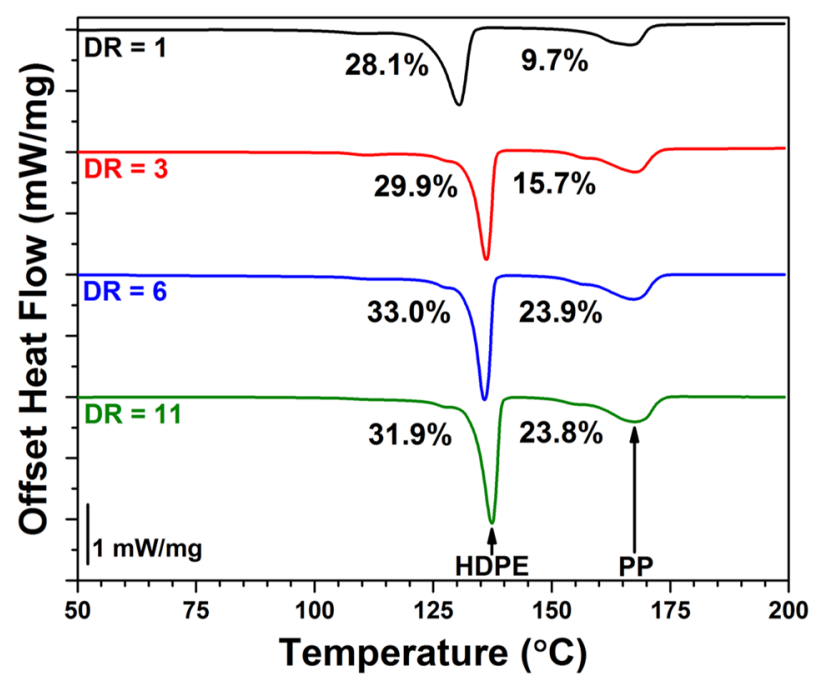

Figure 3. Selected first heating endotherms of the as-extruded composite (DR $=1)$ and drawn (DR $=3,6$, and 11) HDPE/PP/PS composites with HDPE/PP crystallinity of 28.1/9.7\%, 29.9/15.7\%, $33.0 / 23.9 \%$, and $31.9 / 23.8 \%$ respectively.

To determine the impact of orientation within the fiber-like domains, a volume additive model was used to extrapolate the contribution from the fiber-like HDPE and PP domains $\left(E_{F}\right)$ to the overall modulus (Equation 4). The volume fraction of PS ( $\left.V_{P S}\right)$ was taken as $9 \%(0.09)$ from the nominal flow rate during multilayer coextrusion. The modulus of PS drawn under the same conditions at $\mathrm{DR}=1$ and $\mathrm{DR}=11$ was determined to be $1170 \pm 30 \mathrm{MPa}$ and $1210 \pm 80 \mathrm{MPa}$, respectively, suggesting that drawing of the amorphous PS domains induced no change in mechanical behavior. An average value of $1190 \mathrm{MPa}$ was chosen to represent the contribution of PS domains $\left(E_{P S}\right)$ to the overall modulus $\left(E_{T}\right)$. Any enhancements in composite modulus were assigned to an increase in modulus of the PP and HDPE fiber-like domains due to the observed crystalline orientation during uniaxial drawing. (Figures $\mathbf{2 b}$ and $\mathbf{4 c}$ ). 

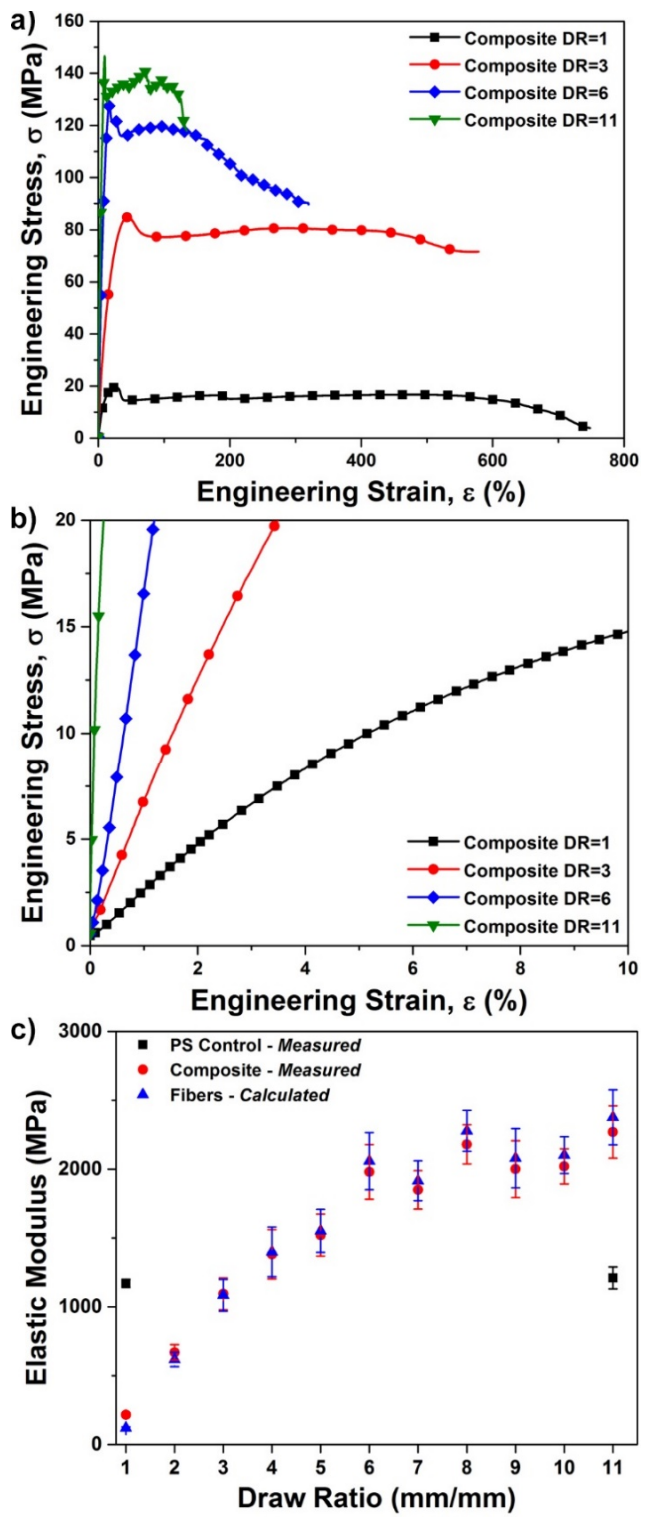

Figure 4. Representative a) full and b) low-strain region stress vs. strain profiles of as-extruded $(D R=1)$ and uniaxially drawn $(D R=3,6$, and 11) HDPE/PP/PS composites; $c)$ measured composite and PS modulus along with calculated effective HDPE/PP fiber-like phase modulus as a function of draw ratio.

\section{Fiber Mat Characterization}

After correlating uniaxial drawing, fiber domain structure, and composite mechanics, we fabricated HDPE/PP fibrous mats by removing the PS matrix via high pressure jet, which simultaneously delaminates and consolidates the fibers into a mat. ${ }^{1} \mathrm{H}$ NMR spectroscopy was utilized to quantify residual PS content after high-pressure water jetting for $1,2.5$, and $5 \mathrm{~min} / \mathrm{cm}^{2}$ (Figures 5a and S2) by monitoring the aromatic proton shift region (6.30-7.20 ppm). The amount of PS diminished with increasing water jet time, and no residual PS was observed after $5 \mathrm{~min} / \mathrm{cm}^{2}$ (Figure 5a). FTIR also provided a qualitative assessment of residual PS content (Figure 5b). Reduced FTIR absorbance peaks at $3100-3000 \mathrm{~cm}^{-1}\left(3026 \mathrm{~cm}^{-1}: \mathrm{C}-\mathrm{H}\right.$ stretching in aromatics) and 
at $1600-1400 \mathrm{~cm}^{-1}$ (1453 and $1493 \mathrm{~cm}^{-1}$ : C-C stretching of the aromatic rings) were indicative of minimal residual PS in the HDPE/PP fiber mixture after delamination.

It was anticipated that delamination of the PS matrix from the composite would be straightforward due its brittleness and small interdiffusion distance (Table S2). However, the simultaneous separation of the HDPE and PP domains was expected to be more challenging due to their higher interdiffusion distance. The efficiency of fiber separation by water jetting was tracked by measuring changes in specific surface area with water jetting time. The specific surface area increased dramatically up to $2.5 \mathrm{~min} / \mathrm{cm}^{2}$ with minimal changes with longer exposure times (Figure 5c). The HDPE/PP fibrous domains were easily separated, which was rationalized by the synthetic approach utilized to manufacture the HDPE and PP resins. Both the HDPE and PP were commercially developed via a Ziegler-Natta polymerization. Polyolefins produced in this way contain amorphous, oligomeric components that tend to migrate to the boundaries during extrusion. [48] These oligomers inhibit the interdiffusion of chains between the HDPE and PP phases, resulting in poor adhesion and the facile fiber separation observed in the HDPE/PP/PS composites. Due to the dual requirements of fiber separation and PS removal, a minimum water jet time of $5 \mathrm{~min} / \mathrm{cm}^{2}$ was required.

Upon optimization of the delamination process to obtain HDPE/PP fiber mats, the fiber size and specific surface area were analyzed as a function of draw ratio (Figure 6). As expected, the width and thickness of the dual-component HDPE/PP fibers decreased with increasing draw ratio from 9 width $\times 3$ thickness $\mu \mathrm{m}(\mathrm{DR}=1)$ to 5 width $\times 0.9$ thickness $\mu \mathrm{m}(\mathrm{DR}=11)$ (Figure 6a, Figure S5, Table 2), confirming affine deformation during uniaxial drawing (Figure S4). [38] The variation in fiber dimensions observed via SEM was attributed to non-uniformity in the fiber cross-section (i.e. the cross-section is not perfectly rectangular). The decreasing fiber dimensions also enhanced the specific surface area by $\sim 3600 \%$, increasing from $0.063 \mathrm{~m}^{2} / \mathrm{g}(\mathrm{DR}=1)$ to 2.32 $\mathrm{m}^{2} / \mathrm{g}(\mathrm{DR}=11)($ Figure 6b, Table 2). 

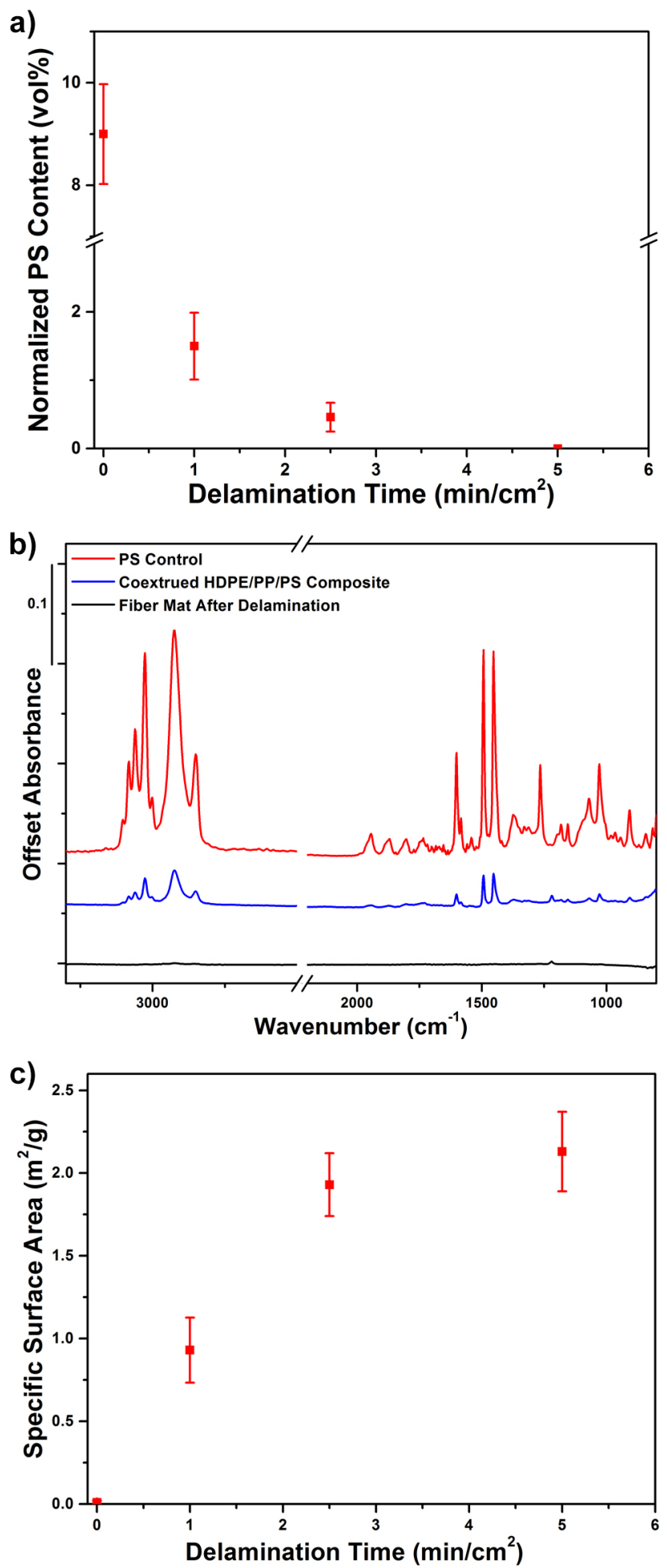

Figure 5: Normalized polystyrene content via NMR (a) and specific surface area (c) of fiber composites as a function of delamination time. FTIR spectra (b) for PS control, coextruded HDPE/PP/PS composites, and fiber mat after delamination for $5 \mathrm{~min} / \mathrm{cm}^{2}$. 

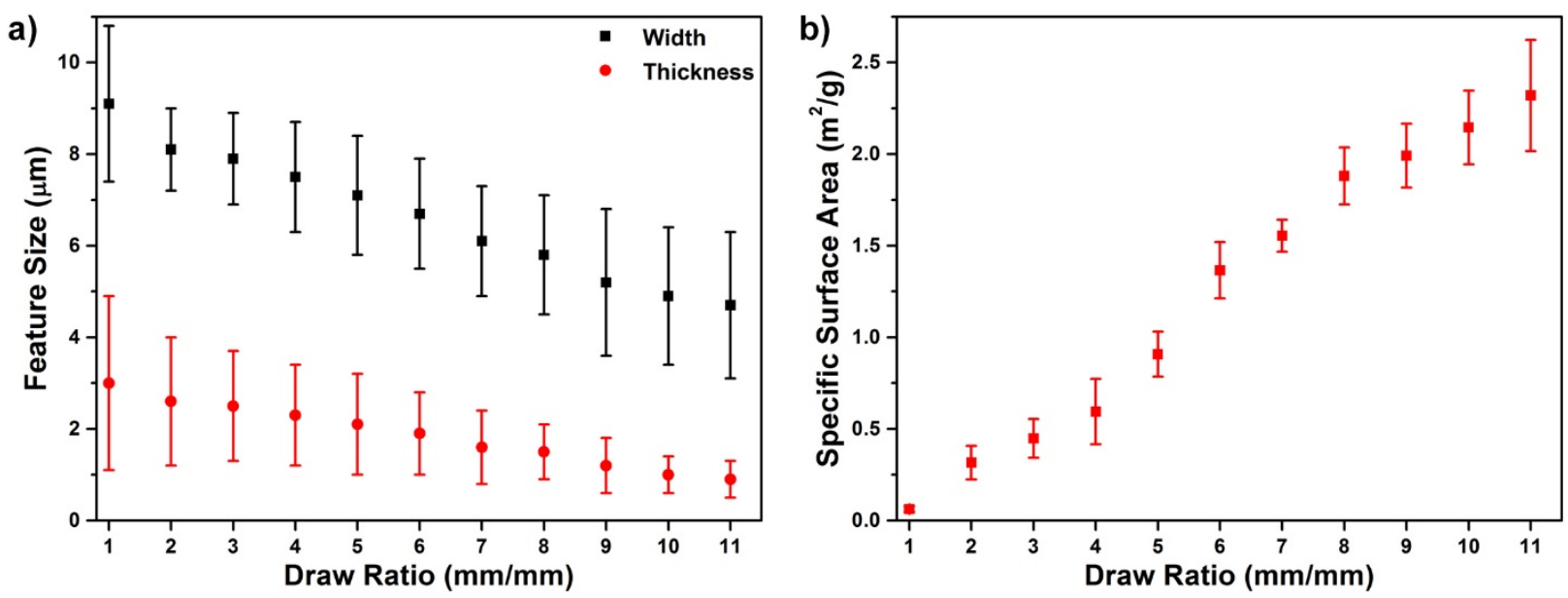

Figure 6: Width and thickness measured via SEM (a) and specific surface area (b) of fibers as a function of draw ratio.

The tunable physical properties of the HDPE/PP/PS composite provided the framework for understanding the structural properties of the consolidated HDPE/PP fiber mat. Extruded mats comprised of HDPE/PP fibers oriented at various DRs were investigated to probe the connection between the mechanical responses of the unseparated HDPE/PP/PS composites to that of the equivalent extruded HDPE/PP fibrous mats. As expected, the elastic modulus of the HDPE/PP mats increased as the DR was varied from $2(1340 \pm 200 \mathrm{MPa})$ to $11(2010 \pm 230 \mathrm{MPa})$ (Table 2, Figure 7). These results are significant in the context of comparison to electrospun PP fibers with a reported modulus on the order of $20 \mathrm{MPa}$ as a non-woven and up to $170 \mathrm{MPa}$ as a single fiber, compared to a modulus of $2160 \mathrm{MPa}$ for the extruded HDPE/PP fiber mat at DR 11. [49] The lowest modulus fiber mats produced via melt coextrusion still have a modulus $650 \%$ higher than an equivalent electrospun mat, which can be attributed to the improved crystallization achieved by melt processing. The crystallinity achieved in electrospun fibers is limited by the rate of nucleation and growth compared to the rate of solvent evaporation in electrospinning and cannot be improved via drawing and orientation; for the electrospun PP fibers, higher boiling point solvents were shown to increase crystallinity and enhance mechanics. However, in the melt state, the extruded polyolefin fibers crystallize more slowly during take-off and crystallinity is further improved via drawing and orientation. The inherent flexibility of the melt coextrusion of ultra-fine fibers provides distinct advantages compared to solvent-based techniques, such as electrospinning, as both the fiber size and mechanical properties can be tuned independently. Process parameters, such as the number of multipliers and take-off method, may be utilized to control the initial size, while the mechanical properties may be tuned via uniform, post-process orientation. 


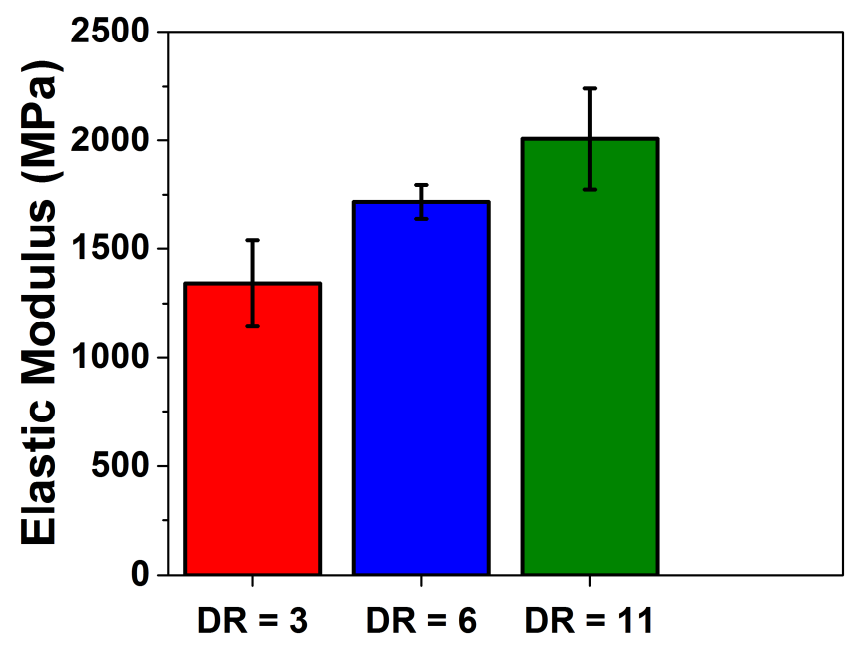

Figure 7: Elastic modulus of fiber mats at $D R=3,6$, and 11 .

Table 2. Properties of calculated effective HDPE/PP fiber-like phase, separated HDPE/PP fibers, and consolidated HDPE/PP mats

\begin{tabular}{|c|c|c|c|c|c|}
\hline & $\begin{array}{c}\text { Calculated } \\
\text { HDPE/PP fibers } \\
\text { from composite }\end{array}$ & \multicolumn{3}{|c|}{ Separated HDPE/PP fibers } & $\begin{array}{c}\text { Consolidated } \\
\text { HDPE/PP mats }\end{array}$ \\
\hline $\begin{array}{c}\text { Draw } \\
\text { Ratio }\end{array}$ & Modulus (MPa) & $\begin{array}{c}\text { Width } \\
(\boldsymbol{\mu m})\end{array}$ & $\begin{array}{c}\text { Thickness } \\
(\boldsymbol{\mu m})\end{array}$ & $\begin{array}{c}\text { Specific surface area } \\
\left(\mathbf{m}^{2} / \mathbf{g}\right)\end{array}$ & Modulus (MPa) \\
\hline 1 & $220 \pm 10$ & $9.1 \pm 1.7$ & $3.0 \pm 1.9$ & $0.06 \pm 0.02$ & N/A \\
\hline 3 & $1090 \pm 120$ & $7.9 \pm 1.0$ & $2.5 \pm 1.2$ & $0.45 \pm 0.11$ & $1340 \pm 200$ \\
\hline 6 & $1980 \pm 200$ & $6.7 \pm 1.2$ & $1.9 \pm 0.9$ & $1.37 \pm 0.15$ & $1720 \pm 80$ \\
\hline 11 & $2270 \pm 190$ & $4.7 \pm 1.6$ & $0.9 \pm 0.4$ & $2.32 \pm 0.30$ & $2010 \pm 230$ \\
\hline
\end{tabular}

\section{CONCLUSION}

We fabricated HDPE/PP/PS composites via melt coextrusion using a recently developed twodimensional multiplication technique. Before the delamination, the fiber composites were uniaxially drawn to various draw ratios ranging from 1 to 11 to systematically examine the mechanical enhancement of the fiber composites as a result of increasing chain orientation with modulus increasing an order of magnitude from $220 \mathrm{MPa}$ at $\mathrm{DR}=1$ to 2270 at $\mathrm{DR}=11$. The structural evolution was examined using both wide- and small-angle X-ray scattering, and correlated well with both DSC and mechanical data, showing a marked improvement in tensile strength and modulus as a result of the increased crystallinity and crystal uniformity imparted by uniaxial drawing. The composites were delaminated using a high pressure water jet, while simultaneously removing the separating layer of PS to obtain a dual-component mixture of rectangular polyolefin fibers. The ability to tune the fiber size $(9 \times 3 \mu \mathrm{m}$ to $5 \times 0.9 \mu \mathrm{m})$ and 
mechanical properties (1310 MPa to $2010 \mathrm{MPa}$ ) of HDPE/PP polyolefin fiber mats makes them ideal for use in many high performance applications, such as high efficiency filtration, nanocomposites, battery separators, and tissue scaffolds, that require tailored mechanics, porosity, and size.

\section{Acknowledgements}

The authors acknowledge funding from the National Science Foundation (NSF) Science and Technology Center (STC) Center for Layered Polymeric Systems (CLiPS) under Grant DMR0423914 and NSF under Grant CMMI- 1335276. In situ x-ray scattering was performed at the Advanced Polymers Beamline (X27C) at the NSLS-I at BNL supported through the U.S. Department of Energy, Office of Science, Office of Basic Energy Sciences, under Contract DEAC02-98CH10886.

\section{References}

[1] Witter, D. IBISWorld Industry Report 32521: Plastic \& Resin Manufacturing in the US; Industry Report; IBISWorld Inc., 2015.

[2] Vasile, C., Ed. Handbook of Polyolefins, 2nd ed.; CRC Press, 2000.

[3] Barhate, R. S.; Ramakrishna, S. Nanofibrous filtering media: Filtration problems and solutions from tiny materials. Journal of Membrane Science 2007, 296, 1-8.

[4] Cho, D.; Naydich, A.; Frey, M. W.; Joo, Y. L. Further improvement of air filtration efficiency of cellulose filters coated with nanofibers via inclusion of electrostatically active nanoparticles. Polymer 2013, 54, 2364-2472.

[5] Leung, W. W.-F.; Hung, C.-H.; Yuen, P.-T. Effect of face velocity, nanofiber packing density and thickness on filtration performance of filters with nanofibers coated on a substrate. Separation and Purification Technology 2010, 71, 30-37.

[6] Grafe, T.; Graham, K. Polymeric nanofibers and nanofiber webs: a new class of nonwovens. Int. Nonwovens J. 2003, 12 (1), 51-55.

[7] Larrondo, L.; Manley, R. S. J. Electrostatic spinning from fiber melts. I. Experimental observations on fiber formation and properties. J. Polym. Sci.: Polym. Phys. Ed. 1981, 19 (6), 909-920.

[8] Larrondo, L.; Manley, R. S. J. Electrostatic spinning from fiber melts. II. Examination of the flow field in an electrically driven jet. J. Polym. Sci.: Polym. Phys. Ed. 1981, 19 (6), 921-932.

[9] Larrondo, L.; Manley, R. S. J. Electrostatic spinning from fiber melts. III. Electrostatic deformation of a pendant drop of a polymer melt. J. Polym. Sci.: Polym. Phys. Ed. 1981, 19 (6), 933-940.

[10] Kim, B.; Park, H.; Lee, S. H.; Sigmund, W. M. Poly(acrylic acid) nanofibers by electrospinning. Mater. Lett. 2005, 59 (7), 829-832. 
[11] Mo, X. M.; Xu, C. Y.; Kotaki, M.; Ramakrishna, S. Electrospun P(LLA-CL) nanofiber: a biomimetic extracellular matrix for smooth muscle cell and endothelial cell proliferation. Biomater. 2004, 25 (10), 1883-1890.

[12] Sonseca, A.; Peponi, L.; Sahuquillo, O.; Kenny, J. M.; Gimenez, E. Electrospinning of biodegradable polylactide/hydroxyapatite nanofibers: Study on the morphology, crystallinity structure and thermal stability. Polym. Deg. Stab. 2012, 97 (10), 2052-2059.

[13] Zander, N. E.; Orlicki, J. A.; Rawlett, A. M.; Beebe, T. P. J. Electrospun polycaprolactone scaffolds with tailored porosity using two approaches for enhanced cellular infiltration. $J$. Mater. Sci. Mater. Med. 2013, 24 (1), 179-187.

[14] Haghi, A. K.; Akbari, M. Trends in electrospinning of natural nanofibers. Phys. Stat. Sol. 2007, 204 (6), 1830-1834.

[15] Xu, S.; Poirier, G.; Yao, N. Fabrication and piezoelectric property of PMN-PT nanofibers. Nano Energy 2012, 1 (4), 602-607.

[16] Lee, B. L.; Jeon, H.; Wang, A.; Yan, Z.; Yu, J.; Grigoropoulos, C.; Li, S. Femtosecond laser ablation enhances cell infiltration into three-dimensional electrospun scaffolds. Acta Biomater. 2012, 8 (7), 2648-2658.

[17] Thoppey, N. M.; Gorga, R. E.; Bochinski, J. R.; Clarke, L. I. Effect of Solution Parameters on Spontaneous Jet Formation and Throughput in Edge Electrospinning from a FluidFilled Bowl. Macromolecules 2012, 45 (16), 6527-6537.

[18] Zhao, Z.; Li, J.; Yuan, X.; Li, X.; Zhang, Y.; Sheng, J. Preparation and Properties of Electrospun Poly(Vinylidene Fluroide) Membranes. J. Appl. Polym. Sci. 2005, 97 (2), 466474.

[19] Zuo, W.; Zhu, M.; Yang, W.; Yu, H.; Chen, Y.; Zhang, Y. Experimental study on relationship between jet instability and formation of beaded fibers during electrospinning. Polym. Eng. Sci. 2005, 45 (5), 704-709.

[20] Sill, T. J.; von Recum, H. A. Electrospinning: Applications in drug delivery and tissue engineering. Biomater. 2008, 29 (13), 1989-2006.

[21] Cho, D.; Zhou, H.; Cho, Y.; Audus, D.; Joo, Y. L. Structural properties and superhydrophobicity of electrospun polypropylene fibers from solution and melt. Polymer 2010, 51, 6005-6012.

[22] Lee, K.-H.; Ohsawa, O.; Watanabe, K.; Kim, I.-S.; Givens, S. R.; Chase, B.; Rabolt, J. F. Electrospinning of Syndiotactic Polypropylene from a Polymer Solution at Ambient Temperatures. Macromolecules 2009, 42, 5215-5218.

[23] Givens, S. R.; Gardner, K. H.; Rabolt, J. F.; Chase, D. B. High-Temperature Electrospinning of Polyethylene Microfibers from Solution. Macromolecules 2007, 40, 608-610.

[24] Ellison, C. J.; Phatak, A.; Giles, D. W.; Macosko, C. W.; Bates, F. S. Melt blown nanofibers: Fiber diameter distributions and onset of fiber breakup. Polymer 2007, 48 (11), 3306-3316. 
[25] Wang, J.; Langhe, D.; Ponting, M.; Wnek, G. E.; Korley, L. T. J.; Baer, E. Manufacturing of polymer continuous nanofibers using a novel co-extrusion and multiplication technique. Polymer 2014, 55 (2), 673-685.

[26] Badrossamay, M. R.; McIlwee, H. A.; Goss, J. A.; Parker, K. K. Nanofiber Assembly by Rotary Jet-Spinning. Nano Lett. 2010, 10 (6), 2257-2261.

[27] Benavides, R. E.; Jana, S. C.; Reneker, D. H. Nanofibers from Scalable Gas Jet Process. ACS Macro Lett. 2012, 1 (8), 1032-1036.

[28] Jordan, A. M.; Korley, L. T. J. Toward a Tunable Fibrous Scaffold: Structural Development during Uniaxial Drawing of Coextruded Poly( $\varepsilon$-caprolactone) Fibers. Macromolecules 2015, 48 (8), 2614-2627.

[29] Kim, S. E.; Wang, J.; Jordan, A.; Korley, L. T. J.; Baer, E.; Pokorski, J. Surface Modification of Melt Extruded Poly(-caprolactone Nanofibers: Toward a New Scalable Biomaterial Scaffold. ACS Macro Lett. 2014, 3 (6), 585-589.

[30] Kim, S. E.; J., D. W.; E., C. H.; Advincula, A. A.; Pokorski, J. K. Multifunctional and Spatially Controlled Bioconjugation to Melt Coextruded Nanofibers. Polym. Chem. 2015, $6(31), 5683-5692$.

[31] Kim, S. E.; Zhang, C.; Advincula, A. A.; Baer, E.; Pokorski, J. K. Protein and Bacterial Antifouling of Melt Coextruded Nanofiber Mats. ACS Appl. Mater. Interfaces 2016, 8 (14), 8928-8938.

[32] Kim, S.-E.; Harker, E. C.; De Leon, A. C.; Advincula, R. C.; Pokorski, J. K. Coextruded, Aligned, and Gradient-Modified Poly( $\varepsilon$-caprolactone) Fibers as Platforms for Neural Growth. Biomacromolecules 2015, 16 (3), 860-867.

[33] Burt, T. M.; Keum, J.; Hiltner, A.; Baer, E.; Korley, L. T. J. Confinement of Elastomeric Block Copolymers via Forced Assembly Coextrusion. ACS Appl. Mater. Interfaces 2011, 3 (12), 4804-4811.

[34] Burt, T. M.; Jordan, A. M.; Korley, L. T. J. Toward Anisotropic Materials via Forced Assembly Coextrusion. ACS Appl. Mater. Interfaces 2010, 4 (10), 5155-5161.

[35] Burt, T. M.; Jordan, A. M.; Korley, L. T. J. Investigating Interfacial Contributions on the Layer-Thickness-Dependent Mechanical Response of Confined Self-Assembly via Forced Assembly. Macromol. Chem. Phys. 2013, 214 (8), 873-881.

[36] Burt, T. M.; Monemian, S.; Jordan, A. M.; Korley, L. T. J. Thin film confinement of a spherical block copolymer via forced assembly co-extrusion. Soft Matter 2013, 9 (17), 4381-4385.

[37] Ponting, M.; Burt, T. M.; Korley, L. T.; Andrews, J.; Hiltner, A.; Baer, E. Gradient Multilayer Films by Forced Assembly Coextrusion. Industrial and Engineering Chemistry Research 2010, 49 (23), 12111-12118.

[38] Lamnawar, K.; Zhang, H.; Maazouz, A. Coextrusion of Multilayer Structures, Interfacial Phenomena. In Encyclopedia Of Polymer Science and Technology; John Wiley \& Sons, Inc., 2013. 
[39] Jordan, A. M.; Lenart, W. R.; Carr, J. M.; Baer, E.; Korley, L. T. J. Structural Evolution during Mechanical Deformation in High-Barrier PVDF-TFE/PET Multilayer Films Using in-situ X-ray Techniques. ACS Appl. Mater. Interfaces 2014, 6 (6), 3987-3994.

[40] Brunauer, S.; Emmett, P. H.; Teller, E. Adsorption of Gases in Multimolecular Layers. $J$. Am. Chem. Soc. 1938, 60 (2), 309-319.

Magalhaes, A. M. L.; Borggreve, R. J. M. Contribution of the Crazing Process to the

[41] Toughness of Rubber-Modified Polystyrene. Macromolecules 1995, 28 (17), 5841-5851.

[42] Wilchinsky, Z. W. Measurement of Orientation in Polypropylene Film. J. Appl. Phys. 1960, 31 (11), 1969-1972.

[43] Wilchinsky, Z. W. Determination of orientation of the crystalline and amorphous phases in polyethylene by X-ray diffraction. J. Polym. Sci. A-2 Polym. Phys. 1968, 6 (1), 281-288.

[44] Wunderlich, B.; Cormier, C. M. Heat of fusion of polyethylene. J. Polym. Sci. Part A-2: Polym. Phys. 1967, 5 (5), 987-988.

[45] Weeks, J. J. Melting Temperature and Change of Lamellar Thickness with Time for Bulk Polyethylene. Journal of Research of the National Bureau of Standards - A. Physics and Chemistry 1963, 67A (5), 441-451.

[46] Androsch, R.; Wunderlich, B. Heat of Fusio of the Local Equilibrium of Melting of Isotactic Polypropylene. Macromolecules 2001, 34 (23), 8384-8387.

[47] Kamal, M. R.; Feng, L. Distributions of Crystal Size from DSC Melting Traces for Polyethylenes. The Canadian Journal of Chemical Engineering 2004, 82, 1239-1251.

[48] Chaffin, K. A.; Knutsen, J. S.; Brant, P.; Bates, F. S. High-Strength Welds in Metallocene Polypropylene/Polyethylene Laminates. Science 2000, 288, 2187-2190.

[49] Watanabe, K.; Nakamura, T.; Kim, B.-S.; Kim, I.-S. Effect of organic solvent on morphology and mechanical properties of electrospun syndiotactic polypropylene nanofibers. Polymer Bulletin 2011, 67 (9), 2025-2033.

[50] Jordan, A. M.; Viswanath, V.; Kim, S.-E.; Pokorski, J. K.; Korley, L. T. J. Processing and Surface Modification of Polymer Nanofibers for Biological Scaffolds: a Review, J. Mater. Chem. B, 2016, DOI: 10.1039/c6tb01303a. 